lait provenant de la traite du matin. L'étable s'étant augmentée d'un animal ayant mis bas courant novembre, la quantité de lait fourni par les 4 vaches, pour l'ensemble de la traite, a été de 14 litres.

Les résultats de l'analyse furent les suivants :

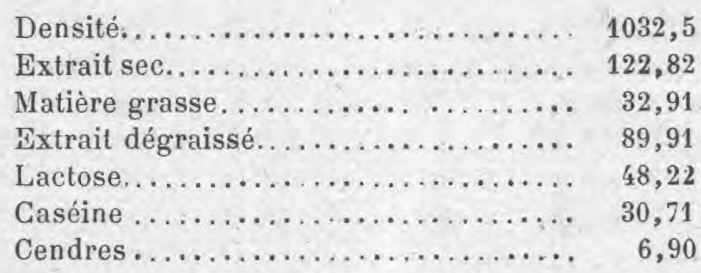

Les animaux recevaient, à ce moment, une alimentation mixte comprenant du fourrage sec ajouté aux collets de betteraves.

L'extrait dégraissé a atteint la moyenne, mais le taux de matière grasse est encore très bas.

Deux conclusions s'imposent à la suite de cette observation :

$1^{\circ}$ Certains aliments aqueux, pauvres en substances nutritives, abaissent la teneur du lait en matière grasse, sans augmenter la quantité de lait fourni.

$2^{\circ}$ En l'absence d'échantillon de comparaison, l'expert doit se montrer aussi réservé en matière de mouillage qu'en matière d'écrémage et se garder de conclure affirmativement.

\title{
LA LUTTE GONTRE LA DISETTE DU LAIT PENDANT LA GUERRE 1914-1918 (suite),
}

\author{
par le Dr A.-J.-J. VANDEVELDE,
}

Professeur de chimie. générale à l'Institut Agronomique Supérieur de l'Etat, Directeur du Laboratoire chimique et bactériologique de la Ville de Gand, Directeur de l'Institut Supérieur des Industries de fermentation.

\section{II. - Gentralisation et pasteurisation du lait.}

La Commission scientifique d'études pour la lutte contre la disette du lait décida, le 22 février 1916, de centraliser le lait nécessaire aux œuvres communales, et d'en faire la haute pasteurisation au moyen d'appareils que j'avais proposés, et dont je donne plus loin la description; les détails en sont indiqués dans une brochure (1) publiée en 1916 et qui fut vendue au profit des CEuvres de la Goutte de Lait.

(1) Ch. Duguesne, R. Neiryngk el A.-J.-J. Vandevelde. - Le Lait pasteurisé, dispositif nouveau de pasteurisation rationnelle pour la distribution dans les OEuvres de la protection de l'Enfance. Gand, 1916. 
En voici les points les plus importants :

Le récipient à lait utilisé dans l'usine de la haute pasteurisation du lait, n'est autre chose que le ballon Pasteur agrandi et adapté au travail spécial qu'il doit fournir.

Aussitôt que possible, après la traite, le lait est filtré et introduit dans des bidons en fer étamé, de 20 litres, munis d'un couvercle fermant hermétiquement, du modèle utilisé dans les laiteries. Les bidons sont remplis presque complètement; entre la surface supérieure du liquide et la paroi inférieure convexe du couvercle, il n'y a qu'un espace de 2 à 5 $\mathrm{cm}$. Le couvercle lui-même porte deux tubes en étain ou en fer étamé de $1 \mathrm{~cm}$. de diamètre intérieur ; l'un de ces tubes est recourbé et taillé en biseau pour éviter pendant l'écoulement du liquide, lors de la distribution, la chûte des poussières et des bactéries atmosphériques dans le lait du bidon; pendant le chauffage, ce tube est fermé à l'aide d'un bouchon de liège. L'autre tube est court, il porte un bouchon muni d'un tube de verre recourbé, l'ouverture extérieure libre atteignant la paroi extérieure concave du couvercle. Le bidon ainsi préparé est recouvert d'un chapeau métallique protecteur en fer blanc (ou en zinc quand le fer blanc fait défaut). Le chapeau est maintenu par des ficelles plombées au bidon lui-même pour empêcher toute ouverture en cours d'expédition, et éviter ainsi toute contamination extérieure par suite de soustraction frauduleuse. Le bidon à lait ainsi modifié est donc en réalité un grand ballon Pasteur adapté à l'usage industriel.

Les appareils sont maintenus au bain de vapeur, de manière à chauffer le lait, pendant 20 minutes, à $100^{\circ} \mathrm{C}$. Il faut alors retirer les bidons des douches de chauffage, les refroidir rapidement dans de l'eau froide courante, puis les maintenir dans une cave fraîche, ou, mieux, dans une glacière jusqu'au moment de la distribution.

Les bidons de lait sont pesés, un échantillon est prélevé de chacun d'eux, et soumis immédiatement à l'essai de fraicheur, soit par le chauffage, soit par le mélange avec de l'alcool, soit par voie alcalimétrique ; de plus, des échantillons multiples sont prélevés et analysés par le Laboratoire communal. L'essii de fraîcheur permet d'éviter que du lait trop acide, ou contenant du colostrum, soit mélangé avec du lait normal et vienne gàter ainsi une grande quantité du précieux aliment.

Le lait reconnu convenable est filtré au travers de toiles métalliques à mailles fines, et réuni dans de grands mélangeurs en tỏle étamée, d'une capacité de 500 à 800 litres, maintenus dans la caveglacière. Le mélange permet d'obtenir du lait de composition moyenne avec des produits de diverses provenances, donc de qualités différentes. 
Le lait des mélangeurs, rendu homogène à l'aide de grandes spatules étamées est introduit, après passage au travers d'un tamis métallique à fines mailles, dans les bidons de distribution déerits précédemment, et qui sont définitivement fermés.Depuis ce moment, jusqu'à celui de la distribution, le lait ne subit plus aucun contact direct avec l'extérieur.

Les appareils de ehauffage consistent en douches chauftées par la vapeur directe et par des serpentins à vapeur, avec soupapes de réglage. Un double fond percé de trous, sur lequel reposent les bidons, protège ce serpentin. La vapeur, sous une pression de 8 atmosphères, circule dans les serpentins et porte l'eau contenue dans les douches à la température d'ébullition au bout d'une dizaine de minutes. L'eau de condensation et la vapeur d'échappement sont dirigées vers un réservoir à eau, de façon à permettre de préparer, sans frais et continuellement, l'eau bouillante nécessaire au nettoyage. Les douches sont recouvertes d'un couvercle en tôle, à charnières avec contrepoids. Un thermomètre fixé sur le réservoir, permet de lire la température intérieure. Quand le thermomètre marque $100^{\circ} \mathrm{C}$, la soupape de commande peut être fermée partiellement, de manière à ne laisser circuler qu'un mince filet de vapeur, ce qui suffit pour maintenir la température de $100^{\circ} \mathrm{C}$. pendant tout le temps de l'opération.

Le lait à $100^{\circ} \mathrm{C}$. émet de la vapeur d'eau qui s'échappe par la tubulure courbe en verre, stérilise, comme s'il s'agissait du ballon Pasteur lui-même, les deux tubulures et l'intérieur du chapeau protecteur, e'est-à-dire toutes les parties internes de l'appareil Une petite quantité de lait s'échappe parfois par cette tubulure, mais elle rentre dans le bidon pendant le refroidissement.

Lors du remplissage, les bidons ont été plombés et ne seront plus ouverts sous aucun prétexte avant la distribution. Pour nous permettre, cependant, de connaitre l'état du lait à l'intérieur des bidons après le chauffage, nous plaçons dans les douches après l'opération, un petit récipient couvert, en fer émaillé, contenant une petite quantité de lait, identique à celui qui est chauffé dans les bidons. L'état de ce lait, après le chauffage, correspond nécessairement à celui qui est contenu dans les bidons fermés.

Un ehauffage de 10 à 20 minutes à $100^{\circ}$ C. stérilise pratiquement le lait et suffit à empècher la fermentation laetique pendant plusieurs jours lorsque le refroidissement se fait dès la pasteurisation, et quand les bidons sont placés dans une cave de conserve suffisamment refroidie.

Dès que le chauffage est terminé, les soupapes d'arrivée de vapeur sont fermées, les couvercles des douches sont retirés et après que la 
vapeur s'est un peu dissipée, les bidons sont enlevés. Il est nécessaire de protéger, pendant cette manipulation, les mains des ouvriers par de gros gants de cuir brut.

Il est indispensable de refroidir sans délai le contenu des bidons, de manière à ramener rapidement la température du lait à une température inférieure à $25^{\circ} \mathrm{C}$. Cette opération s'effectue dans de grandes euves en bois ou en ciment, permettant de receroir plusieurs bidons, dans lesquelles circule un courant continu d'eau froide, réglé tant par le tuyau d'amenée que par des écoulements de trop plein à différents niveaux. On obtient aussi de bons résultats en faisant arriver sur les bidons de l'eau en minces filets, un peu au-dessus du col, de manière à éviter l'entrée de l'eau sous les chapeaux de protection. On réalise ainsi une grande économie d'eau.

Le séjour dans les appareils de refroidissement est de 30 à 45 minutes, selon la température de l'eau, laquelle doit toujours être inférieure à $15^{\circ} \mathrm{C}$.; après quoi les bidons sont prêts pour l'expédition et conservés dans la cave-glacière. Le refroidissement immédiat après le ehauflage lent ou insuffisant, permet aux quelques bactéries que la chaleur peut ne pas avoir détruites, de reprendre leur activité, de se multiplier, et de provoquer dans le lait la fermentation lactique ou souvent même putridè(Bacillus subtilis).

A l'arrivée au local de distribution, les dames vérifient les plombs et accusent la réception par un reçu imprimé sur lequel elles sont priées de joindre leurs observations. L'examen de toutes les informations permet au service d'éviter les défauts signalés et de se perfectionner dans la mesure du possible. De petites chaises basculantes, en bois, d'un modèle spécial, et peintes en blanc, permettent une distribution facile, sans perte.

Les bidons sont ramenés, le plus tôt possible, au local de pasteurisation pour y subir le nettoyage. Le couvercle-fermeture muni de ses tubulures est un objet délicat. La couche d'étain qui recouvre la tôle a une épaisseur limitée. Tout nettoyage à la brosse dure, avec ou sans carbonate de sodium est à rejeter, il faut employer exclusivement l'eau chaude et la vapeur sous pression.

A la canalisation de vapeur est raccordée une canalisation secondaire, munie de gros tubes verticaux fournissant le jet de vapeur nécessaire au nettoyage et à la désinfection de l'intérieur des bidons et des tubes plus étroits, avec dispositif de rotation dans le plan vertical pour le nettoyage des tubulures fixées sur le couvercle.

Les bidons sont ainsi journellement nettoyés et désinfectés, et toutes les mesures nécessaires doivent être prises pour éviler la contamination du lait pendant les transvasements qu'il subit depuis son arrivée jusqu'à son introduction dans les bidons : matériel, manipu- 
lateurs, parquets doivent être d'une propreté irréprochable. L'eau, la vapeur, le carbonate de sodium, le formol, le permanganate, doivent entretenir autour du lait, sans le toucher, l'atmosphère pure, indispensable, qu'il trouve si rarement dans les laiteries industrielles.

Jusqu'à présent, le lait pasteurisé se vend en bouteilles fermées, ce qui constitue, quand le chauffage a été bien conduit, un procédé de choix ; ou bien le lait est traité par masses dans des appareils continus et déversés, après refroidissement, dans des récipients non stérilisés, traitement qui, au point de vue hygiénique, est fort aléatoire. Dans le nouveau procédé, le lait est pasteurisé, transporté et débité sans changer de récipient. Le lait d'un bidon incomplètement vidé, peut être facilement conservé jusqu'au lendemain, dans un local frais, à condition de fermer le tube de déversement an moyen d'un tampon d'ouate flambé.

La perte du lait est réduite au minimum, le chauffage en bidon fermé conduit à une perte bien inférieure à $1 \mathrm{p}$. 100, la production peut atteindre 1.000 litres par heure, puisque les appareils utilisés permettent le maniement de 21 bidons de 20 litres par demi-heure.

L'entretien du matériel se réduit exclusivement à la vérification des fermetures hermétiques en caoutchoue et au renouvellement des bouchons. D'après l'expérience acquise, les bandes de caoutchouc de fermeture de certains bidons, doivent être renouvelées tous les deux ou trois mois. Cette matière fut souvent rare et de qualité inférieure; les essais que nous avons faits avec diverses substances nous ont amenés à employer le papier ou la pâte de papier pour fixer hermétiquement les couvercles sur les bidons. Le cuir et les cordes de différentes fibres manquent d'élasticité et ne fournissent pas une fermeture hermétique.

Enfin, le lait lui-mème fut placé sous un contrôle permanent; dans une période de disette, lorsque les fraudes et les détournements deviennent une chose courante, il convient plus que jamais de prendre des mesures pour assurer la salubrité des denrées. Les enfants reçurent ainsi du lait réellement pasteurisé, et présentant une composition bien déterminée. Le contrôle est l'une des opérations les plus délicates dans un service comme celui qui nous occupe ; celui qui le pratique doit se placer, sans souci et sans crainte, au-dessus de toutes les influences qui l'entourent, il doit avoir recours à des méthodes sûres et être prudent dans ses conclusions. Il faut donc insister sur l'importance de cette partie du travail, dont nous donnons ici un court aperçu. Le degré de fraicheur se détermine par alcalimétrie, à l'aide d'une solution décime-normale d'hydroxyde de sodium. Le lait frais normal exige 15 à $16 \mathrm{~cm}^{3}$ de cette solution par 
$100 \mathrm{~cm}^{3}$, pour obtenir la neutralisation. Pour des quantités voisines de $20 \mathrm{~cm}^{3}$, le lait est de fraicheur suspecte, l'acidité ne peut dépasser

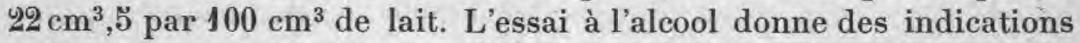
préeieuses : un mélange à parties égales de lait et d'alcool éthylique neutre à 70 p. 100 ne peut donner aucune coagulation quand le lait est suffisamment frais.

L'essai à l'alcool est rapide et se prête très bien à l'examen de nombreux échantillons; il permet, en même temps de déceler la présence de lait colostral.

La lutte contre les souillures fut le souci constant de l'institution. Tout le lait qui arrive est systématiquement vérifié : les souillures du lait qui consistent spécialement en souillures d'étables, sont parfois exagérées ; elles se dosent par comparaison avec l'échelletype, en filtrant le lait sur les disques d'ouate, elles ont atteint d'ailleurs rarement 140 milligr. par litre. Des objets variés ont été retenus au tamisage: vers, limaces, araignées, boutons, débris de paille, de bois et d'étoffes, morceaux de rideaux, ceintures avec boucle, épingles à cheveux, crochets de bottines, lunettes..., de quoi satisfaire les plus difficiles.

Ces objets ont été exposés dans le local de réception avec les noms des fournisseurs dont ils proviennent, et cette exposition permanente, recueillie depuis au Laboratoire communal, a eu une très heureuse influence sur l'état de propreté du lait fourni.

On falsifie le lait par écrémage, c'est-à-dire par enlèvement de graisse, - par mouillage, c'est-à-dire par addition d'eau, - par addition d'antiseptiques. La recherche de la falsification, nécessite une analyse chimique complète; la détermination du poids spécifique et le dosage de la graisse selon le procédé Gerber donnent, en général, des indications suffisantes. Le codex alimenturius néerlandais dit que le lait pasteurisé est du lait qui a été chauffé pendant un temps suffisamment long, entre $65^{\circ}$ et $85^{\circ} \mathrm{C}$. dans les récipients dans lesquels il est lioré à la consommation. Par définition donc, du lait chauffé ou non, contenu dans des récipients servant exclusivement au transport, ne peut être dénommé lait pasteurisé. C'est simplement du lait réinfecté, plus dangereux que le lait cru lui-même.

Liexamen du lait pasteurisé du service s'effectue dans l'usine de pasteurisation et dans les locaux de distribution. Cet examen comporte l'essai de chauffage au cours duquel le lait ne peut devenir bleu qu'après au moins quinze minutes ; le lait non chauffé, ou insuffisamment pasteurisé, donne de suite une coloration bleue.

L'analyse bactériologique complète l'examen, ainsi que la détermination du degré d'acidité.

Les essais chimiques et bactériologiques furent nombreux et 
donnèrent des résultats satisfaisants. L'essai de chauffage a toujours donné des résultats positifs, c'est-à-dire que le lait d'essai additionné des réactifs nécessaires, reste parlaitement blanc. Le nombre des bactéries se maintient à 0 pendant les quatre à cinq premiers jours de culture, pour atteindre à peine 10 à 150 par $\mathrm{cm}^{3}$ de lait après dix jours de culture. Les échantillons recueillis aseptiquement dans les locaux des " Gouttes de Lait », ne coagulèrent généralement pas par le séjour dans le Laboratoire, même six semaines après les prélèvements.

Le service de pasteurisation qui commença, au Laboratoire communal, le 18 avril 1916 , à titre d'essai, fut transporté, le 3 juillet 1916, à l'Institut supérieur des Fermentations; il pasteurisa au début environ 300 litres par jour. Au mois d'octobre de la même année, la production journalière s'élevait à 3.000 litres. Le 31 décembre 1918, la petite usine avait pasteurisé 3 millions $1 / 2 \mathrm{de} \mathrm{kg}$. de lait.

Depuis 1919, le service de pasteurisation est annexé à la Ferme communale où l'on travaille chaque jour un millier de litres de lait produits par la vacherie communale, et destinés à la distribution exclusive des CEuvres communales, Tout récemment, depuis janvier 1921, la distribution est exécutée, en bouteilles, par le service même de la ferme communale.

(A suipre).

\section{BIBLIOGRAPHIE ANALYTIQUE}

\section{$1^{\circ}$ LIVRES.}

Lucius L. van SLXKe. - Modern methods of testing milk and milk products (Méthodes Modernes d'analyse du lait et des produits lactés). Ed. Orange Judd Company, New-York, 1920.

Cet ouvrage dont nous analysons la seconde édition a eu le plus grand succès en Amérique, et l'autorité de son auteur, qui depuis longtemps appartient à la Station Agricole Expérimentale de Geneva, dans l'Etat de NewYork, ne pouvait que lui donner une valeur incontestable.

Ce livre est destiné aux étudiants en laiterie, aux fabricants de beurre et de fromages, aux producteurs de lait, aux fabricants de lail concentré, aux ramasseurs de lait, aux inspecteurs de laiterie, et, en somme, à tous ceux qui désirent avoir sur cette matière des connaissances peut-être un peu sommaires, mais surtout très précises. 